\title{
COMPARISON OF PRECIPITATION EFFECTS IN SPACE-BORNE X- AND KA-BAND SAR IMAGING
}

\author{
A. Danklmayer \\ German Aerospace Center (DLR) \\ Microwaves and Radar Institute \\ Oberpfaffenhofen, Germany
}

\author{
M. Chandra
}

\author{
Chemnitz University of Technology \\ Dept. of Microwave Eng. and Photonics \\ Chemnitz, Germany
}

\begin{abstract}
As the operating frequencies of SAR-systems are increasing, the visible distortions due to precipitation in SAR-images are becoming more frequent. This holds especially for the case of convective rain events. The German space-borne satellite TerraSAR-X has delivered a series of measurement examples, which were used to study precipitation effects in SARimages. Based on these valuable data takes and simultaneous weather radar measurements, a quantitative estimation of precipitation effects in SAR-images is presented.In a further step, an attempt is made to extrapolate the observed effects to systems operating at higher nominal frequency-bands, i.e. Ka-band, being taken under consideration for future SARsystems.
\end{abstract}

Index Terms - Synthetic-aperture radar (SAR), Microwave imaging, Propagation effects, Signal attenuation, Precipitation

\section{INTRODUCTION}

Since 2007, TerraSAR-X is ready to deliver outstanding data, day and night, and during almost all weather conditions. Microwave SAR imaging at X-band frequencies is sometimes affected by propagation effects due to heavy rain, as can be seen in Fig. 1. The physical processes involved are

- reflection at the precipitation volume

- attenuation through the precipitation volume

- change of the surface due to the wetness and for watersurfaces due to the impinging rain drops or due to the influence by the wind

Today, a considerable body of literature exists on propagation effects in SAR imaging and how to make use of SAR data to deduce meteorological information [1][2][3].

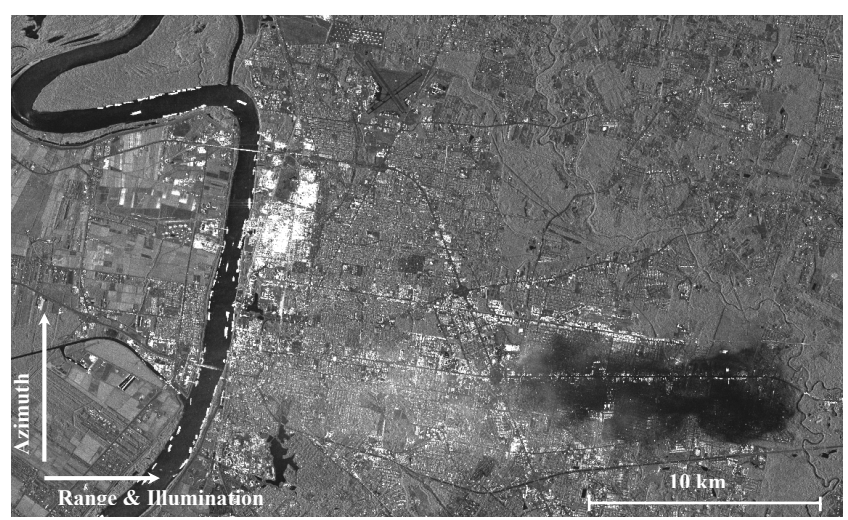

Fig. 1. An example of a SAR image with rain-cell signatures recorded with TerraSAR-X in the US close to Baton Rouge. The white shading is due to direct reflections from the rain region (shown as volume 'A' in Fig. 2). Whereas the darkly shaded areas are due to rain attenuated (blocked) signals from the ground; this effect is shown as region 'B' in Fig. 2.

\section{ANALYSIS OF DATASETS AND PHYSICAL INTERPRETATION OF ARTEFACTS IN SAR IMAGES DUE TO PRECIPITATION}

A physical interpretation of how rain cells affect SAR images is shown in Fig. 2. The sketch shows an imaging scenario, where the transmitted waves interact with a precipitation cell. It can be observed that area 'A', which corresponds to time instant $\tau_{A}$ in the amplitude/time diagram, is due to strong backscattering from large hydrometeors. Time instant $\tau_{B}$, which corresponds to the region ' $\mathrm{B}$ ' proves that signals have been heavily attenuated. By comparing the dark patch in Fig. 1 and the amplitude/time diagram in Fig. 2, the veil in Fig. 1 corresponds to area ' $A$ ' $\left(\tau_{A}\right)$, and the shadowlike black areas close to this veil in Fig. 1 corresponds to the region 'B' $\left(\tau_{B}\right)$ in Fig. 2. 


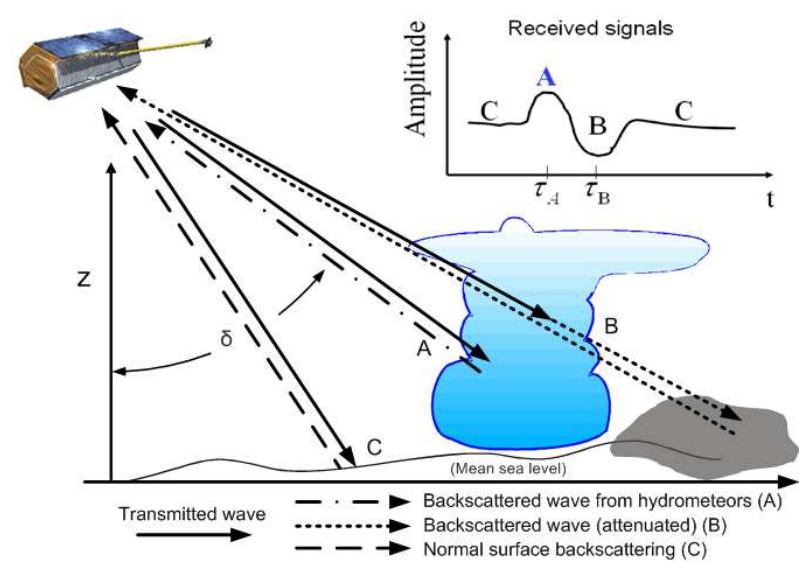

Fig. 2. A physical interpretation of rain cell signatures in SAR-images, as indicated and introduced previously in Fig. 1.

\subsection{Attenuation through rain}

One of the major problems affecting microwave and millimetre wave bands for terrestrial and space-borne radars is the attenuation through rain $[4,5]$. A convenient way to describe the rain intensity is the so called rainfall-rate or rain-rate given in millimetres per hour. This quantity refers to a certain flux of rain towards the surface of the Earth and may be measured e.g. by gauges or weather radars. A widely acceped empirical relation of the form

$$
\gamma(x, t)=a \cdot R^{b}
$$

between specific attenuation $\gamma(x, t)$ and rain rate $R$ is used to calculate the specific attenuation for a given rain rate $[4,6]$. The parameters $a$ and $b$ are dependent on the radio frequency, the raindrop size distribution, the polarization and other factors [6].

After [4], the total attenuation for a given instant of time can be obtained by adding up the specific attenuation along the path of propagation using the following expression

$$
A(t)=\int_{0}^{2 h} \gamma(x, t) d x
$$

where

$$
\begin{array}{ll}
A(t) & \ldots \text { total attenuation for given time instant } \mathrm{t} \\
t & \ldots \text { time } \\
h & \ldots \text { path length } \\
\gamma(x, t) & \ldots \text { specific attenuation } \\
x & \ldots \text { position along the path of propagation }
\end{array}
$$

The specific attenuation along the slant path of propagation has to be known. However, detailed knowledge of the medium through which the signal propagates is rather limited and the temporal and spatial variation of the medium require assumptions and some modelling [7]. In the case of precipitation we may have some idea about the thermodynamic phase

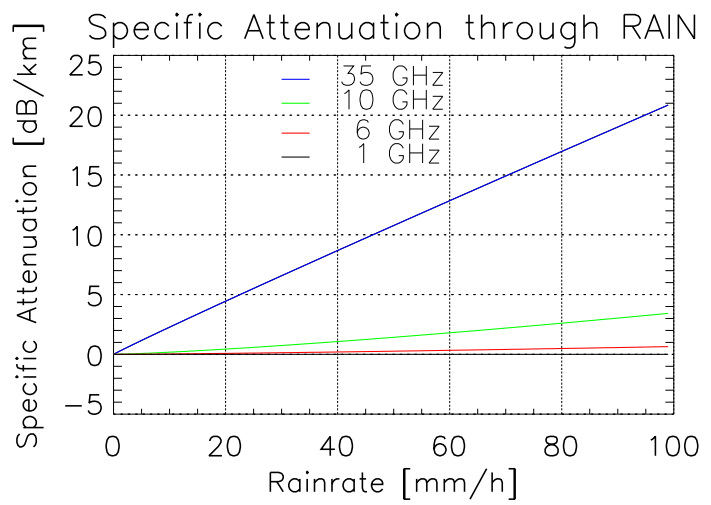

Fig. 3. A plot of the specific attenuation given in units of $\mathrm{dB} / \mathrm{km}$ versus the rain rate in units of $\mathrm{mm} / \mathrm{hr}$ for four different frequencies $(1,6,10$ and $35 \mathrm{GHz})$ after the ITU Model Recommendation 838 (1994).

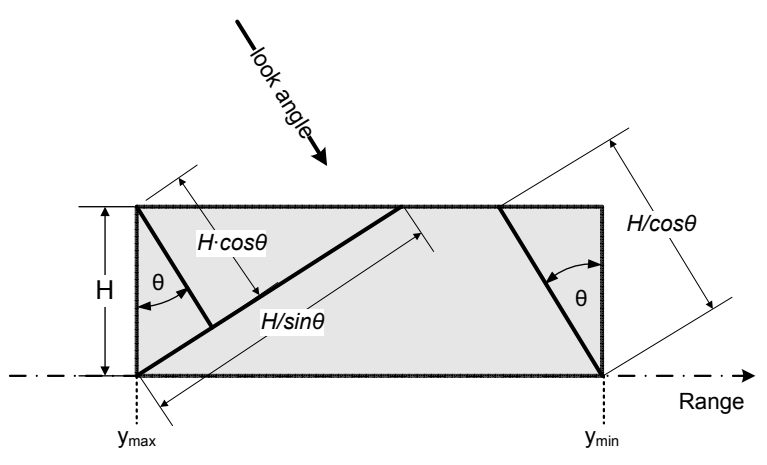

Fig. 4. The structure of an idealised rain cell used for the calculation in Section 2.2.

(ice, water, melting band) but no precise information. Using the calculated values for the specific attenuation in X-band provided in diagram Fig. 3 for a $5 \mathrm{~km}$ long path through a heavy tropical convective rain $(70 \mathrm{~mm} / \mathrm{hr}$ and more $)$ suggests a $20-30 \mathrm{~dB}$ attenuation, which was confirmed by comparing the backscattering coefficient of affected and non-affected region from data takes acquired by TerraSAR-X over Brazilien rain forest.

\subsection{Modelling of the Attenuation under Rain Conditions for X-band and Ka-band}

For the calculations of the rain rate to cause visible attenuation in Ka-band SAR images, a simple model shown in Fig. 4 [8], is used. A reasonable height of $4 \mathrm{~km}$ is assumed, as well as a homogenous rain rate for the modelled cell. The incidence angle of the propagating signals was chosen $30^{\circ}$. With the help of (1) and by using the regression coefficients in Table 1 , the specific attenuation was calculated. These values are given in Table 2. Finally, the values for the two-way path attenuation for different rain rates $(5,50$ and $100 \mathrm{~mm} / \mathrm{hr}$ ) can 
Table 1. Regression coefficients used in (1) and (4)

\begin{tabular}{lcccc}
\hline \multirow{2}{*}{ Frequency } & \multicolumn{5}{c}{ DSD } \\
& $\begin{array}{c}\text { Marshall Pallmer } \\
\mathbf{a}\end{array}$ & $\mathbf{b}$ & $\mathbf{J o s s}$ Thunderstorm \\
& $\mathbf{a}$ & $\mathbf{b}$ \\
\hline X-band (10 GHz) & 0.0136 & 1.15 & 0.0169 & 1.076 \\
Ka-band (35 GHz) & 0.268 & 1.007 & 0.372 & 0.783 \\
\hline
\end{tabular}

Table 2. Specific Attenuation $[d B / \mathrm{km}]$

\begin{tabular}{rcc}
\hline Rainrate & \multicolumn{2}{c}{ Specific Attenuation } \\
{$[\mathbf{m m} / \mathbf{h r}]$} & $10 \mathrm{GHz}$ & $35 \mathrm{GHz}$ \\
\hline 5 & 0.08 & 1.31 \\
50 & 1.22 & 7.95 \\
100 & 2.4 & 13.69 \\
\hline
\end{tabular}

be found in Table 3. As a total two-way attenuation of $25 \mathrm{~dB}$ becomes visible in SAR images in X-band it is of interest which rain rate is necessary to cause such an attenuation for Ka-band $(35 \mathrm{GHz})$ frequencies. To this end, the following equation is applied

$$
\gamma(t)=\frac{A(t)}{2 \cdot \frac{H}{\cos (\theta)}} \quad[\mathrm{dB} / \mathrm{km}]
$$

where the rain rate is found using (1) with the according parameters $a$ and $b$ for Ka-band.

$$
R=\sqrt[b]{\frac{\gamma(t)}{a}}[\mathrm{~mm} / \mathrm{h}]
$$

First calculations using the parameters of the idealised rain cell of $4 \mathrm{~km}$ and using $25 \mathrm{~dB}$ of total two-way attenuation, assuming the Marshall Pallmer Parameters for Ka-band attenuation deliver a rain rate close to $10 \mathrm{~mm} / \mathrm{hr}$. This simple example demonstrates that such rain rates are fully capable to distort Ka-band SAR measurements to a visible extent. In Fig. 5 the range of values for attenuation and rain rate are extendent and the diagram shows the two-way attenuation versus the rain rate for different incidence angles at Kaband. Similar information is provided for X-band frequencies in Fig. 6. The simple assumptions of a homogenous rain cell with constant precipitation may be somehow optimistic, since a melting layer precipitation may severly increase the total path attenuation.

A test case close to New Orleans, US is provided in Fig. 7 and was selected to perform a comparison between two different types of measurements (left: TerraSAR-X; middle: NOAA ground-based weather radar data) and one artificially generated producted (right) based on the modeling approach shown above. The two almost isolated rain cells with reflectivities of up to $50 \mathrm{dBZ}$, depiced in the weatherradar image (middle), are ideally suited to be used for extrapolation and modeling of respective attenuation and backscattering effects for Ka-band. As expected, the attenuation due to rain increases dramatically at Ka-band and therefore the visible artefacts are more pronounced but limited to regions where the rain rate is about $10 \mathrm{~mm} / \mathrm{hr}$. The backscattering at Kaband due to rain is also increased compared to X-band which results in zones of bright veils appearing to the left of the precipitation cells (in the direction towards the radar).
Table 3. Maximum Attenuation for modelled rain cell $[d B]$

\begin{tabular}{rcc}
\hline Rainrate & \multicolumn{2}{c}{ Specific Attenuation } \\
{$[\mathbf{m m} / \mathbf{h r}]$} & $10 \mathrm{GHz}$ & $35 \mathrm{GHz}$ \\
\hline 5 & 0.739 & 12.1 \\
50 & 11.26 & 73.43 \\
100 & 22.17 & 126.46 \\
\hline
\end{tabular}

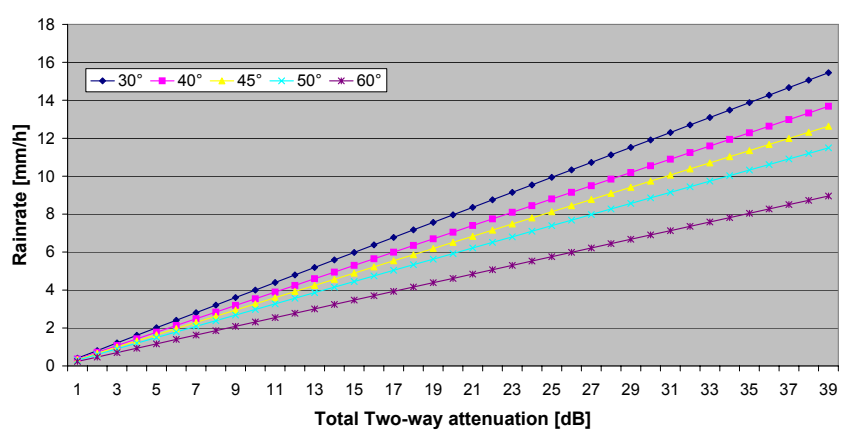

Fig. 5. A diagram of the two-way path attenuation and the corresponding rain rate for the modelled rain cell of $4 \mathrm{~km}$ height (Fig. 4) at Ka-band (35 GHz), for different look angles.

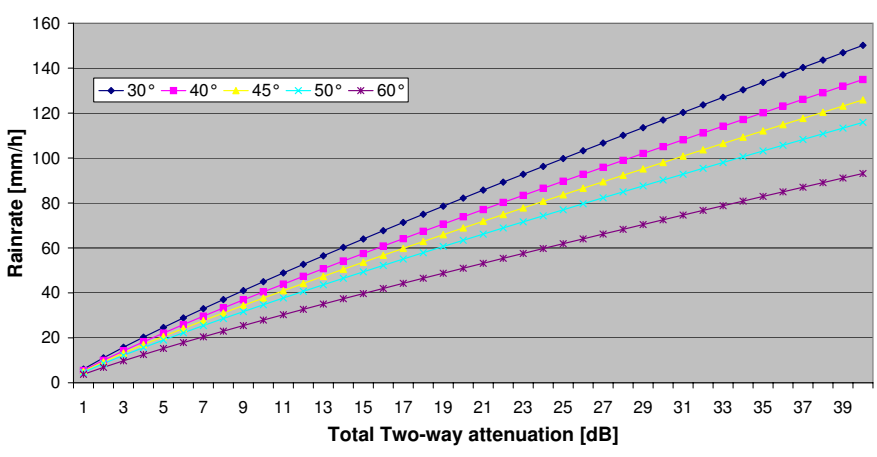

Fig. 6. A diagram of the two-way path attenuation and the corresponding rain rate for the modelled rain cell (Fig. 4) at $\mathrm{X}$-band $(10 \mathrm{GHz})$, for different look angles. 

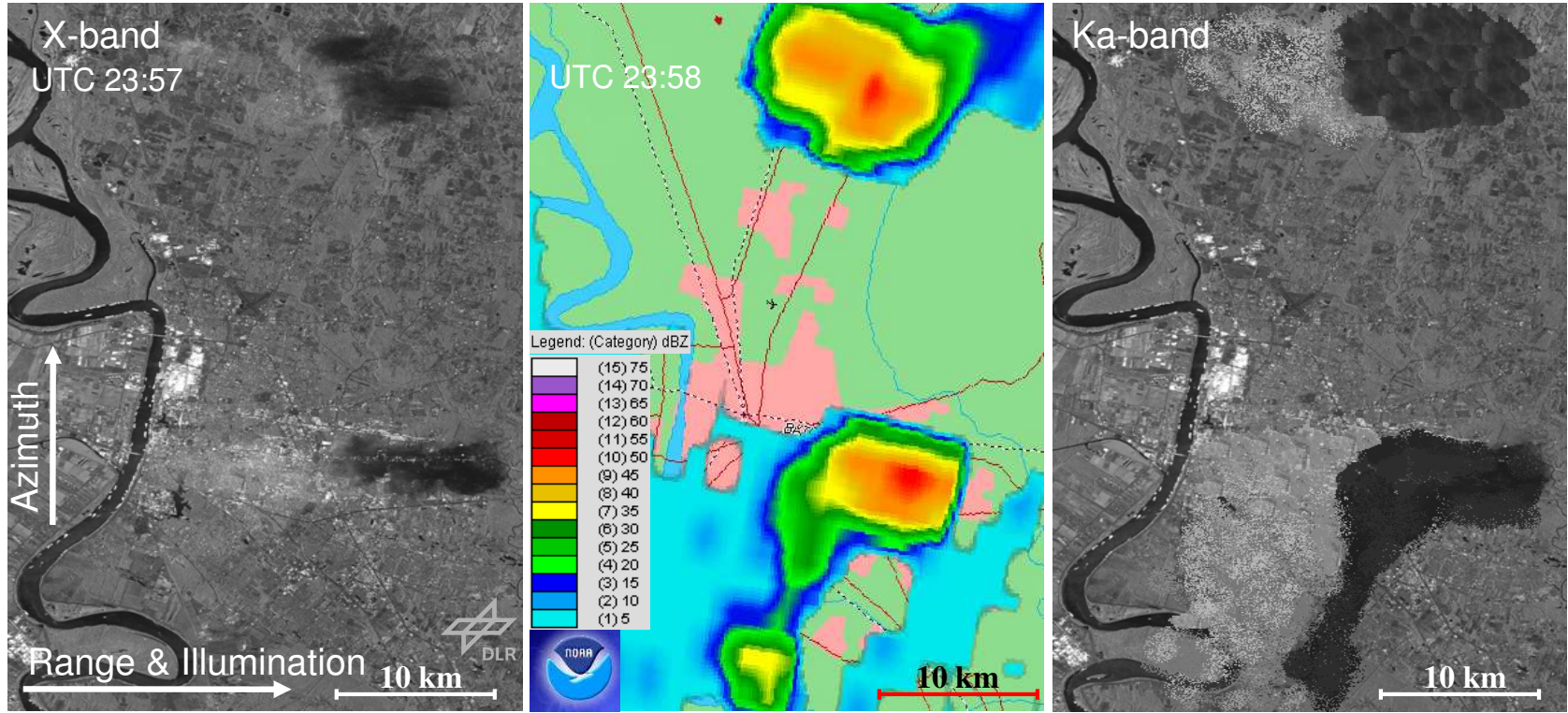

Fig. 7. Depicition and comparison of three different kinds of datasets. In the middle a weather radar image is given, showing a refelectivity map of two almost isolated raincells with reflectivity values up to $50 \mathrm{dBZ}$. To the left a corresponding SAR image measured with TerraSAR-X is provided. Signatures due to precipitation can be clearly identified. To the right, an artificially generated image based on TerraSAR-X data is given and it shows how artefacts at Ka-band may look like. The estimation of the attenuation is based on the modelling provided in Section 2.2.

\section{CONCLUSIONS}

As well known, attenuation due to rain is the dominating effect when considering propagation effects in SAR imaging at higher frequency bands such as X-and Ka-band. For the case of TerraSAR-X only convective precipitation events have the potential to generate visibel artefacts in SAR images. For the Ka-band rain rates of $10 \mathrm{~mm} / \mathrm{h}$ are capable to produce visible artefacts.

\section{Acknowledgements}

This research was partly financed by ESA under contract No. 21876/08/NL/ST/al "Precipitation effects for Ka-band SAR". A. Danklmayer would like to sincerely thank Dipl.Ing. Patrick Tracksdorf for proofreading of the paper.

\section{REFERENCES}

[1] A. Danklmayer, "Propagation effects and polarimetric methods in synthetic aperture radar (SAR) imaging," Ph.D. dissertation, Chemnitz University of Technology, DLR-Report FB-2008-14, Technical University of Chemnitz, 2008.

[2] A. Danklmayer and M. Chandra, "Precipitation induced signatures in SAR images," in Proceedings of Euro- pean Conference on Antennas and Propagation (EuCAP) 2009, Berlin, Germany, 32009.

[3] A. Danklmayer, B. Döring, M. Schwerdt, and M. Chandra, "Assessment of Atmospheric Effects in SAR Images," IEEE Transaction on Geoscience and Remote Sensing, 2009, accepted for publication.

[4] R. K. Crane, Electromagnetic wave propagation through rain. John Wiley and Sons, 1996.

[5] P. Ferrazzoli and G. Schiavon, "Rain-induced modification of SAR performance," Adv. Space Res., vol. 7, no. 11, pp. 269-272, 1987.

[6] R. L. Olsen, D. V. Rogers, and D. B. Hodge, "The $a \cdot R^{b}$ relation in calculation of rain attenuation," IEEE Transactions on Antennas and Propagation, vol. 26, no. 2, pp. 318-329, 1978.

[7] A. W. Doerry, "Atmospheric loss considerations for synthetic aperture radar design and operation," in Proceedings of SPIE, 2004.

[8] C. Melsheimer, Signaturen von Regen in Radaraufnahmen des Meeres. Aachen: Shaker Verlag, 1998. 